\title{
Community Effects on Elderly Health: Evidence from CHARLS National Baseline
}

\author{
James P. Smith ${ }^{\mathrm{a}}$, Meng Tian ${ }^{\mathrm{b}}$, and Yaohui Zhao ${ }^{\mathrm{b}, \mathrm{c}}$ \\ ${ }^{a}$ Chair in Labor Markets and Demographic Studies, The RAND Corporation \\ ${ }^{b}$ National School of Development, Peking University
}

\section{Introduction}

In all societies, the most salient adult outcomes reflect the attributes, choices and behaviors of individuals, their families, friends, and employers. Perhaps equally important are the attributes, opportunities, and constraints that are related to the communities in which people live. This may be especially the case in a country such as China in light of the vast heterogeneity that exists among communities both at a moment of time and in the extent of changes at observed at the community level over time. Communities and villages in China have historically been assigned a central role in the delivery of most essential public services including schools, health care and insurance, and places for social interactions among its residents.

Previous analyses indicate that community-level effects play an unusually large role in explaining adult health outcomes of Chinese residents, often dominating the collective impact of individual level attributes (Strauss et al., 2010; Smith et al., 2012). This result leaves unanswered the more basic question of why and how communities are so important in the Chinese context. Providing some answers to this question is the main motivation of this paper.

One major concern in this research would be how to determine whether the association of community-level characteristics to individual health outcomes is simply due to the fact that people living in communities or villages with worse facilities are those who have lower SES (Socio-Economic Status) or other traits leading to poor health. Evidence of the association between poor individual SES and poor health being "large and pervasive across time and space" is abundant (Smith, 2004). This question can be addressed if both individual/family SES information and community-level characteristics are available.

In this paper, we use a new data source - the Chinese Health and Retirement Longitudinal Survey (CHARLS) - that is nationally representative of those ages 45 and over in the Chinese population in 2011-2012. This data contain detailed demographic, health, and economic information on individuals and families who are part of the study. CHARLS also contains a community-level questionnaire that details current and historical information on the nature of the community, including its economic structure, the provision of basic public

(C) 2013 Elsevier B.V. All rights reserved.

${ }^{\mathrm{C} C}$ orresponding author. yhzhao@ccer.edu.cn.

Publisher's Disclaimer: This is a PDF file of an unedited manuscript that has been accepted for publication. As a service to our customers we are providing this early version of the manuscript. The manuscript will undergo copyediting, typesetting, and review of the resulting proof before it is published in its final citable form. Please note that during the production process errors may be discovered which could affect the content, and all legal disclaimers that apply to the journal pertain. 
services including schools, health care, sanitation, and water supplies. This data allow us to relate the adult life experiences of individuals to the attributes of the places where they have lived. It also allows us to examine the effects of community characteristics while controlling individual/family SES.

This paper is divided into six sections. The next section describes CHARLS data and the main household and community-level variables that will be used in our analysis. Section 3 provides a brief demonstration of the potential importance of geographic/admistrative communities/villages for the health and SES outcomes of Chinese population. Section 4 summarizes the main characteristics of our community-level variables in CHARLS. This summary shows considerable heterogeneity in China on the attributes of communities. Our main empirical findings are contained in section 5 while the final section highlights our main conclusions.

\section{Data: CHARLS}

The China Health and Longitudinal Study (CHARLS) is a nationally representative longitudinal survey of the middle-aged and elderly population $(45+)$ in China along with their spouses, which includes an assessment of the social, economic, and health circumstances of community-residents. ${ }^{1}$ The purpose of CHARLS is to study the main health and economic adjustments to rapid population aging in China. The national baseline survey of CHARLS was conducted between June 2011 and March 2012 on 17,692 respondents. The survey followed strict randomization procedures. At the first stage of sampling, 150 county-level units were randomly chosen with the probability proportional to scale (PPS) from a sampling frame containing all county-level units of China excluding only Tibet. At the second stage, three communities (administrative villages in rural areas or resident committees in urban areas) were randomly chosen with the PPS method from a sampling frame containing all communities in the county-level units. At the third stage, all dwelling units in a community were listed to create a sampling frame following an extensive mapping and listing operation using a software developed by the CHARLS team which utilized Google Earth map images, from which a certain number of dwelling units were randomly chosen. In rare cases where the dwelling contained more than one household with age-eligible individuals, the computer randomly picked one. If a household had more than one age-eligible member, again the computer randomly chose one as the main respondent. Spouses of main respondents were automatically included.

CHARLS respondents will be followed every two years using a face-to-face CAPI interview. CHARLS has been harmonized with leading international research studies in the Health and Retirement Study (HRS) model to ensure adoption of best practice and international comparability of results. CHARLS baseline data include detailed information of respondents and their living spouses. The CHARLS main household questionnaire contains information on basic demographics, family, health status, health care, employment, household economy (income, consumption and wealth). All data are collected by face-toface computer-aided personal interviews (CAPI). Both questionnaire and field procedures were repeatedly tested to ensure high data quality.

The main adult outcome variables include key adult health and SES outcomes. Adult health include self-reported general health status, doctor diagnoses of chronic illnesses, depression, word recall, lifestyle and health behaviors (physical activities, smoking, drinking), subjective expectation of mortality, activities of daily living (ADLs), and instrumental

${ }^{1}$ For a detailed description of the CHARLS survey, see Zhao et al., "Cohort Profile: The China Health and Retirement Longitudinal Study International Journal of Epidemiology. Forthcoming. 
activities of daily living (IADLs). It is worth noting that some health variables, such as hypertension, weight and height, are obtained from health measurements conducted in the field.

Financial dimensions of SES in CHARLS are measured in terms of income, wealth and consumption expenditure. CHARLS separately measures income and assets at the individual level as well as at the household level. CHARLS income components include wage income, self-employment income, agricultural income, pension income and transfer income, where wage income is collected for each of the household members, and transfer income separates government transfers specific to individuals from those to households.

Asset measurements collected at household level include housing, productive assets, financial assets, consumer durables and land. Information on ownership status, value and characteristics of current residence as well as other housing owned by the household are recorded. Deposits and other investments are measured at the individual level, but debts are asked both for respondent and spouse, and for the household.

Household expenditures are collected in CHARLS since the literature has shown that expenditure can be a better welfare measures than income in developing countries (Strauss and Thomas, 2008). Consumption items are collected at weekly, monthly and yearly frequencies respectively to minimize recall bias. Food expenditure is collected on weekly basis. It includes expenditures on dining out, food bought from market and values of homeproduced food consumed. Food expenditures induced by inviting guests for important events are collected to better reflect household food expenditure per capita in a normal week. Monthly-based expenditures are those usually spent each month, including fees for utilities, nannies, communications, etc. Yearly-based items record expenditures occurred occasionally in a year, including traveling, expenditures on durables, and education and training fees.

In addition to the household survey, a detailed community-level questionnaire was formulated. As detailed in the section that follows, this community questionnaire focuses on important infrastructure available in the community, plus the availability of health facilities, prices of goods and services that are also often used by the middle aged and the elderly.

\section{Geographical Disparities of Health and SES Outcomes in China}

Although research interest in the determinants of population health in both developed and developing countries has surged over the past few decades, one issue that needs greater attention is understanding effects of community characteristics on health and SES outcomes of residents and how these effects then translate into large health and SES disparities across geographical boundaries. To illustrate, Murray et al. (2006) found that the gap between the highest and lowest life expectancies across different race-county combinations in the United States is 35 years. Similarly, Rosenzweig (1982) found strong influence of community infrastructure and climate factors on child morality and fertility in Colombia.

China can be an important experimental ground for examining community effects because with the rigid household registration system (hukou), China has traditionally restricted geographical mobility so that the distribution of people across communities especially in the age groups considered here is more exogenous especially in the age groups we consider in this paper. Despite the Chinese government's constant interventions aimed at improving social environmental factors that could produce health benefits and reduce disparities associated with geography, there apparently still exist exceedingly wide gaps across communities and villages. 
Table 1 presents the distribution of health and SES variations across two types of geographical and administrative units in China-counties and communities. For each type of geographic unit for each health and SES outcome, Table 1 lists the mean and median values alongside values at the top and bottom 5\% of geographic units as our measures of heterogeneity. With the not surprising exception of household ln PCE which exhibits positive skewness, mean and median values tend to be quite close indicating close to normal distributions in these outcomes.

The first panel in Table 1 shows the distribution of health and SES outcomes of different counties, which in China are the 6-digit administrative divisions below the level of province and municipalities, but above the geographic level of townships and communities/villages. These include city districts. The geographic disparities displayed are nothing short of staggering. The prevalence of poor or very poor SRH for the top 5 percentile counties is less than one third of the bottom 5 percentile counties. Similarly, the percent of the population 45 years old or above having ADL (activities of daily living) difficulties is 9 times larger in the bottom 5 percentile counties than the first 5 percentile counties. For IADL (instrumental activities of daily living) difficulties, this ratio is more than 6 times larger. Depression scores for the worst 5 percentile counties are 7.4 points larger than the best 5 percentile counties, and prevalence of hypertension is less than half of that for the better off counties. People from the top 5 percentile counties can be $8 \mathrm{~kg}$ heavier, and 10 centimeters taller than those who are from the bottom 5 percentile counties. They also tend to have 7.5 more years of schooling. Finally, the income gap between the top 5 percentile and the bottom 5 percentile counties is around 6 times.

If we look at those statistics across communities/villages, the lowest level of government administration, instead of counties, disparities by geography are even starker. To illustrate with just a few examples from the bottom half of Table 1, the worst $5 \%$ of communities have hypertension rates of $64 \%$ compared to $19 \%$ for the healthiest $5 \%$ of communities. The comparison for IADLS would be $47 \%$ for the worst off $5 \%$ and $3 \%$ for the best off. The magnitude of these health and SES disparities across geography raises the obvious question of what factors underlie those gaps, and what measures can be taken to improve individual health and SES at the community level based on these findings.

Besides the geographical differences of counties and communities, Hukou system has important healthcare implications for residents with different household registration. For example, rural areas received far less social expenditure than those received by cities. Due to this fact, the government focus more on preventative measures than curative healthcare measures, and a large number of rural health workers (bare-foot doctors) are trained to function in place of the regular doctors (Zhang and Kanbur, 2005). Appendix Table 1 provides a detailed description of health and SES variations at county/community level by different hukou registration (Urban/Rural). For example, the incidence of poor self-reported health is only $9 \%$ in better-off urban counties, $14 \%$ in better-off rural counties, $36 \%$ in worst-off urban counties, and 50\% in worst-off rural counties. There is a significant gap between the average urban and rural health and SES levels. The inequality of health between the better-off and worst off areas is more salient in rural areas than in urban areas (eg: a CESD score gap of less than 6 in urban counties, and 7.5 in rural counties). The inequality of SES between the better-off and worst off areas, however is more noticeable in urban areas than in rural areas as the better-off urban counties/communities is very well developed.

\section{Characteristics of Communities in CHARLS}

The CHARLS community-level survey asks informed officials/personnel in the community about characteristics and histories of the communities in which CHARLS respondents 
reside. There are 450 communities/villages in the CHARLS survey, 213 of which are in urban areas and 237 in rural places. Dimensions of these communities used in this research involve aspects of the environment; both those made by human intervention and those associated with nature that might plausibly affect the health of community residents.

Questions on the current infrastructure and public facilities of communities include types of drinking water used (tap water, well, pool, river and lakes, cellar, and spring); types of cooking fuel that is used in the village (hay, coal, marsh gas, natural gas, liquefied petroleum gas (LPG), and other), what type of waste disposal system is present (moved away by truck, buried in village, burned, dumped into nearby river, or no management), the main forms of toilet system (in-house, out-house or open air and for each type with or without flushing water). As the details in this list imply, many aspects of village infrastructure may imply elevated health risks for community inhabitants.

One issue that arises in characterizing communities and assessing their effects on CHARLS respondents concerns that extent of geographic mobility of CHARLS respondents over their lives. Table 2 shows the extent of mobility of CHARLS respondents since their birth. The main mobility portrait that comes from Table 2 is clear. CHARLS respondents who are by design ages 45 and over were relatively immobile ${ }^{2}$. At the time of the CHARLS interview, almost half of CHARLS respondents lived in the same community or village in which they were born. Even among those who did migrate after birth, they did not venture far. Threequarters of those who did move lived in another village in the same county. Thus, close to nine in ten CHARLS respondents were living in the same county in which they were born and only $6 \%$ were living in a different province in which they were born. This lack of geographic mobility reflects the fact that the age groups in the CHARLS sample were adults largely before the period of extensive migration in China.

Population mobility was restricted mainly because of the existing hukou registration system. The Hukou system was implemented initially in Chinese cities in 1951 and then extended comprehensively in 1955 . With this system, any change of residence has to be officially sanctioned. Collectivization of agricultural land gives no room for outsiders to obtain land thus restricted migration. De-collectivization of agricultural production freed farmers to conduct nonfarm activities, but the land was still collectively owned. Even today, unless in areas with mass exodus of the labor force, we rarely see migrant farm households farm the land. Central job allocations in cities makes it very difficult for anyone without hukou to move. The decentralization in the job market is a recent phenomenon, which ushered in this great migration as we see today, even without an explicit hukou policy change.

Not surprisingly, mobility is much higher among CHARLS respondents currently living in the urban areas. Compared to 58\% in the rural areas, 34\% of urban residents currently live in the same community in which they were born. But even in urban areas lifetime mobility of respondents is relatively small-79\% of urban CHARLS respondents live in the same county where they were born and less than one in ten are living in a different province.

As mentioned above, environmental risks on which we focus in this paper include types of water, how waste is managed types of toilet facilities, and cooking fuels. Table 3.A lists the distribution of those environmental hazards for the full CHARLS sample as well as urban and rural areas separately. In each category following the name of the individual risk, we

\footnotetext{
${ }^{2}$ Due to survey design, we can only capture mobility using migrants moving into the communities. We don't have the characteristics of the communities the migrants are from. If the migrant moved into a community with worse environment and SES, the higher mortality rate of local residents may cause mobility to be overestimated. If the migrant moved into a community with better environment and SES, the higher mortality rate of migrants may cause mobility to be underestimated. There is no definitive evidence as to which case is more likely to happen
} 
also indicate in Table 3.A the grouping of items that we will employ in our modeling below. Not surprisingly, these types of environmental hazards are far more common in rural places in China compared to urban places.

To illustrate, tap water is considered the safest type of water, and a little more than half of CHARLS communities obtain their water from tap water. Three-quarters of rural communities do not have tap water compared to one-fifth of urban places. The second most common type of water used is well water as $55 \%$ of rural communities and $17 \%$ of urban communities use that form. A very similar overall distribution and urban-rural divide exists for type of waste management. Two-thirds of rural communities do not manage their waste at all compared to only $14 \%$ in urban areas. Among those communities who do manage their waste, moving away by truck is the most common method but again with a large urban (81\%)-rural (17\%) difference.

Turning next to the toileting system used, only $43 \%$ of CHARLS communities have indoor toilets, a fraction that falls to less than one in five in rural places. The most common forms of toileting in rural areas are open air fields which characterizes about $60 \%$ of rural areas in China. Some types of cooking fuels as they become airborne can also be a significant health hazard. CHARLS community respondents were asked to categorize cooking fuel usage into six types-hay, coal, marsh gas, natural gas, liquefied Petroleum Gas (LPG) and other. Table 3.A shows that hay is the most common form in the rural areas and LPG in the urban areas, but there is a great deal of heterogeneity of types in both rural and urban communities in China.

Given China's geographic expanse and its varied landscape, differences in natural environmental endowments across communities/villages in China are also quite remarkable. Table 3.B displays the variability by highlighting in the upper panel variation across communities in temperature, rainy days, and snowy days. According to CHARLS 20112012 community data, Northern China can have severe winters with temperatures reaching 30 centigrade below zero, while southern part has moderate winters above 6 centigrade. China's climate is mainly dominated by dry seasons and wet monsoons. The east coastal areas or some basin areas may have around 130 days of rain per year, while some of the inland places may have as few as a week's rain per year. The average snowy days are 8 days per year nationwide. Provinces in the South may have no snow at all, but Northern provinces could stay with the snowy weather for as long as a month.

The bottom panel in Table 3.B displays differences in topography by urban and rural places. Topography type distributions can be varied between urban and rural areas. In general, the country mostly consists of large areas of plain as well as vast mountainous and hilly areas. Urban communities are twice more likely than rural villages to locate on the plain, while rural villages are about twice more likely to be in the hilly and mountainous area. For plateau and basin, which are the least commonly seen topography types in China, there is no significant difference in urban/rural divisions.

\section{Empirical Findings}

The goal of this study is to explore the importance of community characteristics in predicting individual levels of health and SES. We group predictors of individual health into the following three factors: social-economic environments, physical environments, and individual characteristics and behaviors. ${ }^{3}$ Our measures of SES are seen to be affected by a

3WHO: http://www.who.int/hia/evidence/doh/en/ 
similar set of factors. We start with a simplified version of Grossman's model (1972) as follows:

$$
\begin{gathered}
Y_{i}=\beta_{0}+\beta_{1} \text { Demo }_{i}+\beta_{2} \text { indSES }_{i}+\beta_{3} h h S E S_{h}+\beta_{4} \text { comX } \\
j \\
\varepsilon_{i}=\beta_{j}+\beta_{5} i n d H B_{i}+\varepsilon_{i}
\end{gathered}
$$

$Y_{i}$ includes two types of outcome variables. The first are measures of individual health such as general health status on the conventional five point scale from very good to very poor, activities of daily living (ADLs), instrumental activities of daily living (IADLs), CES-D scores for depression, hypertension, weight, and height. People are defined as hypertensive if either a doctor told them they were hypertensive or they measured over the convention diagnostic thresholds of 140/90. Both weight (in kilograms) and height (in centimeters) are obtained by direct measurement during the interview. The second set of variables in $\mathrm{Y}_{\mathrm{i}}$ is our two SES measures-years of schooling and natural log of household expenditure per capita.

On the right hand side of equation (1) are three types of variables. The first consist of standard demographics such as age, sex, marital status and residential area type $\left(D^{2} \mathrm{oro}_{i}\right)$ which are well known to be related to health and individual SES. In the health outcome equations, we also include individual SES measured as three dummy variables for educational accomplishments, illiterate (omitted category), primary school education, middle school education and above, and household resources indexed by the natural log of household expenditure per capita.

The second type of individual level variables (ind $H B$ ) includes whether one smokes (ever smoke or currently smoking) and whether one drinks alcohol more than once a month (ever or currently drinking).

The third set of variables form the center of our analytical focus since they all relate to the attributes of communities in which people live. Our community-level variables capture both measured and unmeasured attributes of the community that may alter individual health and SES. Our measured community-level variables start with the average economic status of the community which we proxy by the mean log community PCE of residents in the community (excluding the respondent household). To capture effects of physical environments, we bring in a set of community features $\left(\operatorname{com} X_{j}\right)$ which measure type of water, toileting, waste pollution, and cooking fuel used in the community as well as some physical features of the community - temperature, rainy and snowy days, and type of terrain of the community (hill, mountainous, plateau, or basin). Finally, the unmeasured aspects of the community are denoted by the error term $\varepsilon_{j}$ which captures other relevant factors unique to the community. Equation (1) specifies the error term as composed by two factors: individual idiosyncratic errors, and county level errors from differences in physical environments of the communities.

We begin in Table 4 by presenting our baseline OLS results for a model that does not include any measured or unmeasured aspects of the community so that these baseline results can be compared with subsequent models that take into account aspects of the community. Thus, models in Table 4 only include personal attributes and behaviors. Our findings are quite consistent with the existing literature (for example, Winkleby et al., 1992; Adler et al., 1994; Smith and Kington, 1997; Strauss et al., 2010).

Examine first the demographic variables. Not surprisingly, health tends to deteriorate with age even though most of the effects are non-linear, reflecting both life-cycle health declines and improvements in health as we move toward more recent Chinese birth cohorts (who 
must necessarily be younger). The decline in Ln PCE and especially education with age most likely primarily reflect cohort effects of improving levels over time in China. Similarly, men are generally healthier than women in all health domains in Table 4 and on average have 2.8 more years of schooling than Chinese women do. However, Chinese men and women share similar levels of per Capita Expenditures as this is measured at the household rather than the individual level.

Married adults are healthier than non-married adults in virtually all health domains (Waite and Gallagher, 2000), and have higher levels of education but fewer economic resources since our measure of Ln PCE is defined on a per capita basis and there are likely economies of scale. Chinese urban residents are in better self-reported health, have fewer IADLs, are less depressed and have higher education and Ln household PCE. Urban residents do have higher rates of hypertension, perhaps because they are more likely to be overweight as is also demonstrated in Table 4.

As documented in several studies both in a Chinese (Strauss et al., 2010) and other contexts (Smith, 1999), there is a strong positive education gradient in health models in Table 4 so that higher levels of education are associated with better health. Similarly, higher levels of economic resources either at the household or community-level are positively associated with better health outcomes. Our two health behavior variables - smoking and drinking yield conventional results. Those Chinese respondents (mostly Chinese men) who have ever smoked or drank are less healthy. The positive association of good health with being a current smoker most likely reflects a selection effect of quitters from both behaviors doing so for health reasons.

As mentioned above, models in Table 4 considered only effects of individual attributes, largely ignoring any role played by the physical and natural environment. By re-estimating the same model as in Table 4 with a set of county fixed effects, our first attempt to introduce these community factors is agnostic about what particular features of the community may matter. Counties are the first level of geographic aggregation above the individual communities themselves in the CHARLS data. There are 150 Chinese counties represented in the CHARLS data. These results are presented in Table 5.

Not surprisingly, the $\mathrm{R}^{2}$ in Table 5 are higher than in Table 4 reflecting the collective explanatory power of community-level effects at the county level. The estimated coefficients in Table 5 are similar to our Table 4 coefficients without county fixed effects although generally slightly smaller in magnitude. The main exception to that generalization involves the two community-level variables in these models- urban and community Ln PCE. The coefficients of those two variables were uniformly reduced in magnitude, occasionally by a sizable amount. To use but two examples, controlling for county fixed-effects, the effect of being an urban resident on reducing the likelihood of having poor or very poor selfreported health dropped from 5.6\% to 3.0\% and the effect of depression (CESD) was substantially reduced from -0.819 in Table 4 to -0.308 in Table 5. Similarly, the statistically significant negative effect of community Ln PCE in Table 4 is not statistically significant when county-fixed effects are added. The other exception to the rule of a small reduction in estimated coefficients concerns the health behavior variables which are also much smaller in magnitude in Table 5.

These comparisons of Tables 4 and 5 strongly suggest that there are important attributes of communities that are being left out of our baseline models that are significantly affecting health and SES outcomes. The effects of these omitted community variables are being picked up by the already included community variables (urban and community Ln PCE) and individual health behaviors and are therefore exaggerating their effects. 
Therefore, we introduce our third set of models which include specific variables depicting the physical environments in which people live. Table 6 presents results that include variables indicating community/village physical environment such as drinking water types, waste managements, toileting types and cooking fuel types. In our analysis, we separate water into three types-surface water, underground water, and tap water. Tap water, which is the reference group in our models, is generally thought to be the healthiest form since in China it is often filtered and attempts are made to eliminate bacteria and other health contaminants. The ranking of the other two sources of water is ambiguous a prori as both sources are subject to different types and sources of contamination. For example, surface water is subject to sources of contamination from the air and earth while underground water is subject to possible contamination from buried industrial and minerals.

Our estimates ${ }^{4}$ in Table 6 indicate that using surface water (river, pool, snow, rain) increases the likelihood of worse health especially compared to the tap water reference group. The probability that self- reported health is poor or very poor is increased by $6.0 \%$, and having any IADL difficulties is raised by $5.3 \%$. Surface water usage increases the CES-D (Depression) score by approximately 0.6 points. Surface water also has negative impacts on years of schooling.

Underground water also has negative health effects, albeit smaller in some health outcomes than for surface water. Compared to tap water, underground water is strongly associated with higher depression, lower height and less schooling. The height effect is particularly interesting in light of the lack of mobility of most of the CHARLS respondents documented above. These residents most likely were exposed to similarly unhealthy water when they were children, the years in which their height may have most susceptible to external health environment. Collectively our results in Table 6 do indicate that the quality of water is an important aspect of the community that can have negative impacts on individual health and SES.

We also separated waste management into three groups-waste moved away from the locality by trucks (the reference group), non-management of waste, and other waste management such as burying in the local area, burning, or disposal in rivers and lakes. Compared to moving away by trucks which should be the healthiest of the waste management methods, the other two types of waste management tend to be associated with worse health and SES with non-management not surprisingly the worse method. If the community/village did not manage waste, the likelihood of residents having poor/very poor SRH would increase 3.1 percentage points, CES-D score would increase about 0.43 point, height would reduce by four tenths of a centimeter, and years of schooling would reduce by about 0.20 years. Other managements are not very significant, but slightly increase the probability of ADLs or IADLS.

Methods of toileting are divided into three groups- inside toilet with water (the reference group), outside toileting with water that may be shared with others in the community, and toileting without water. The reference group should be the healthiest and the other two forms have different advantages and disadvantages in terms of health of residents. The estimates in Table 6 indicate that toilets without water have the worse consequences for health outcomes with statistically significant negative health consequences for SRH, both IADLS and ADLS, being overweight, and hypertension. Bad toileting conditions may increase chances for

\footnotetext{
${ }^{4}$ There are possible problems with causality inference from our models. Better community facilities could be results of active demand for public goods. For example, tap water installation could be results of severely polluted water source. It is also possible that some observables left out in the error are correlated with the community characteristics and health/SES, and SES itself could be affected by community characteristics. Therefore, it is better to regard our estimates as predicted associations between community characteristics and individual health and SES outcomes.
} 
falling and reduce frequencies to visit. Our results also show people having toilets without water tend to be taller; this might be because people are taller in dry areas where water is not easily and cheaply accessible. Our two SES measures- years of schooling and Ln household PCE- are also smaller.

We also investigated possible impacts associated with different types of cooking fuels used in the communities. Cooking fuel usage was divided into five types. The first is natural gas and/or liquid petroleum gas, which serves as our reference group since these are thought to have less negative health effects in part as they have a smaller impact on the quality of indoor air. In contrast, two alternative and very common cooking fuels in China are hay and coal, both of which are widely used especially in rural areas. Cooking fuels such as coals and hays are usually considered as the primary source of indoor pollution in China (Zhang et al., 2007). Hay is often burned in the open air and affects the lungs as of course does coal. Marsh gas is a new biological fuel source promoted by the government as a cleaner source of cooking fuel.

As expected, our results show that hay and coal have the largest negative effects on health and SES. When these are used as the cooking fuel, self-reported health is worse, there are more ADLs and IADLs difficulties, depression is higher, people are shorter, and education is lower by about a year. Other types of fuels have strong negative impacts on health and SES.

In addition to these human-made environmental hazards, we also include in these models measures of the natural environment in which people live. These measures include for the last year minimum and maximum temperature in a locality, the number of rainy and number of snowy days in the community. It is harder for middle aged and elderly people to cope with extreme temperature. Rainy and snowy days affect elderly health in terms of reinforcing chronic pains, preventing exercise and access to immediate treatment, reducing social activities. Literature has found in Japan, disability-free life expectancy is strongly associated with snowy and rainy days (Noda et. al 2011). Extreme cold weather is known to be associated with health risks of poor circulation, respiratory problems and accidents. ${ }^{5}$ This may be a particularly important problem in developing countries where protection from the cold such as indoor heating is not widely available. We do find that increases in the lowest temperature do lead to improvements in self-reported health. We find that higher temperature is positively associated with having ADL/IADL difficulties and Depression, and reduces height slightly.

More rainy and snowy days in a year are associated with people being more depressed while snowy days increase both IADLS and ADLS, most likely due to people with some type of impairment having more difficulty dealing with the snow for walking. A big difference between rainy and snowy dates is that rainy days are associated with lower height and schooling while snowy days are the opposite. The existing literature finds more rainfalls during gestation and early childhood adversely affect people's height because they are more likely to have parasitic infections (Godoy et al. 2008)

Mountain areas are generally very bad for individual health and SES, especially in reducing people's heights. The remoteness associated with living in mountains and to a lesser extent hills most likely means less access to health care and the diet may be worse since there is less access to markets and nutritious foods.

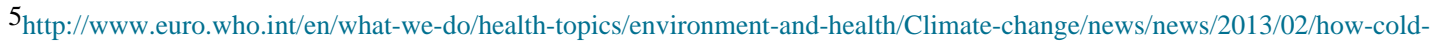
weather-affects-health/adverse-health-effects-of-exposure-to-cold.
} 
In the models in Table 6, we found coefficients of urban resident variables have been reduced to an extent that they are even much smaller than our fixed-effect model, not to mention some of them became insignificant. The community/village variables we introduced into Table 3 explained a very large part of the urban/rural differences. A somewhat similar pattern can be found with the community PCE coefficients. An interesting fact would be adding those variables reinforces the already significant effects of household PCE, that is, given the community physical environment, individual resources become more important to individual health. Given the vast urban-rural difference in China (Sicular et. al, 2006), it might be inadequate to only assume that the "urban" indicator only affects the intercept but not the slopes as specified in our models. We then extend our analysis to a Blinder-Oaxaca decomposition of the rural-urban gap in health and SES outcomes (See on-line Appendix Table 3). We categorize the factors into 7 groups: human-made environments, climate, topology, demographics, education, household expenditure, and health behaviors, and examined the contributions from each of these groups to rural-urban gap in health and SES. We found that differences in endowments, especially community characteristics such as human-made environments, climate and topology can explain most of the urban-rural differences, except for hypertension (eg: 67\% for SRH poor/very poor, $83 \%$ for education). Compared to other characteristics such as demographics and health behavior, endowments of community facilities and natural environments played a more important role in urbanrural divide.

\section{Conclusions}

There is increasing interest in neighborhood or area effects on health and individual development. Our research on this question in the context of China in this paper addresses two questions: whether characteristics of community/village people where people live matter, and why they matter. In this research, we merged information on socioeconomic, infrastructure, and climatic-geographic characteristics of 450 communities with over 17,000 individual-household records from China Health and Retirement Longitudinal Study 20112012 National Baseline. Estimated relationships are reported between individual health and SES and the characteristics of communities in which respondents live.

Our statistical results indicate that community/village characteristics have strong associations with individual health and SES. Controlling for county fixed-effects, effects of community level variables such as urban/rural residence and Ln community PCE on health and SES outcomes were uniformly reduced in magnitude, indicating that there are important attributes of communities that are being left out in the simple OLS models commonly estimated. When we include specific variables depicting the physical community/village environments in which people live, the coefficients of urban resident variable have been reduced even more than those in the fixed-effect models, and some of the significance is gone as well.

CHARLS 2011-2012 data provide us a rich set of information on community/village physical environmental characteristics, such as water managements, waste managements, toileting system, cooking fuels and also records on temperature, rainfalls, snowfalls and landscapes. We found that using surface water increases the likelihood of worse health compared to tap water and even underground water. Compared to moving away by trucks, non-management of waste, and other management such as dump in local site or nearby water body are associated with worse health and SES outcomes.

Toileting system without water has the worst influence on individual health and education achievements. As a developing country, China adopts miscellaneous types of fuels for cooking and heating. Due to its extensive and frequent usage, cooking fuel is considered the 
primary household pollution source. Our results show that hay and coal have the largest negative effect on health and SES outcomes. Extreme weather conditions such as very low or very high temperatures and long periods of rainfalls/snowfalls cause people to be more depressed, and face severe difficulties in ADL or IADL and other negative health conditions. Local landscapes also affect individual health and SES outcomes as mountainous and hilly areas exacerbate individual health status and SES outcomes.

Our research investigated measured community/village characteristics in CHARLS and their relation to individual health and SES outcomes. However, CHARLS does not include all aspects of people's life in the community/village that may be important. For example, domestic animals might be a risk factor to individual health as they may carry bacteria or insects. Similarly, residents of rural area may suffer from exposure to farming chemicals. Additional research is needed concerning these and other community-level factors that may significantly impact individual health and SES in China and other countries of the world.

\section{Supplementary Material}

Refer to Web version on PubMed Central for supplementary material.

\section{Acknowledgments}

This research was supported by grants from the National Institute on Aging and Natural Science Foundation of China.

\section{References}

Adler, Nancy E.; Boyce, T.; Chesney, M.; Cohen, S.; Folkman, S.; Kahn, RL.; Syme, SL. Socioeconomic Status and Health: The Challenge of the Gradient. American Phychologist. Jan; 1994 49(1):15-24.

Godoy, et al. Comprehensive mass-spectrometry-based proteome quantification of haploid versus diploid yeast. Nature. 2008; 455:1251-1254. [PubMed: 18820680]

Hu, Yisong; Smith, James P.; Strauss, John; Yang, Gonghuan; Zhao, Yaohui. Cohort Profile: The China Health and Retirement Longitudinal Study. International Journal of Epidemiology. advance access published November 9, 2012. 10.1093/ije/dys168

Murray C, Kulkarni S, Michaud C, Tomijima N, Bulzacchelli M, Iandiorio T, Ezzati M. Eight Americas: Investigating Mortality Disparities across Races, Counties, and Race-Counties in the United States. PLOS Medicine. 3(9):e260.10.1371/journal.pmed.0030260 [PubMed: 16968116]

Noda T, Ojima T, Hashimoto S, Kawado M, Seko R, Kato M, Murata C, et al. SP3-40 Disability-free life expectancy and related factors in Japan. Journal of Epidemiology and Community Health. 2011; 65(Suppl 1):A419-A419.

Robert, Stephanie A. Community-Level Socioeconomic Status Effects on Adult Health. Journal of Health and Social Behavior. Mar; 1998 39(1):18-37. [PubMed: 9575702]

Rosenzweig MR. Child Mortality and Fertility in Colombia: Individual and Community Effects. Health Policy and Education. Mar; 1982 2(3-4):305-348. [PubMed: 10256651]

Sastry, Narayan. Community Characteristics, Individual and Household Attributes, and Child Survival in Brazil. Demography. May; 1996 33(2):211-229. [PubMed: 8827166]

Sicular, Terry; Yue, Ximing; Gustafsson, Bjorn; Li, Shi. Research Paper No 2006/135. UNU-Wider; Helsinki: The Urban-Rural Income Gap and Inequality in China.

Smith JP, Kington Raynard. Demographic and Economic Correlates of Health in Old Age. Demography. Feb; 1997 34(1):159-170. [PubMed: 9074837]

Smith JP, Shen Yan, Strauss John, Yang Zhe, Zhao Yaohui. The Effects of Childhood Health on Adult Health and SES in China. Economic Development and Cultural Change. Fall;2012 61(1):127-156. [PubMed: 23539191] 
Strauss, John; Lei, Xiaoyan; Park, Albert; Shen, Yan; Smith, James P.; Yang, Zhe; Zhao, Yaohui. Health Outcomes and Socio-Economic Status among the Elderly in China: Evidence from the CHARLS Pilot. Journal of Population Ageing. Dec; 2010 3(3-4):111-142. [PubMed: 23539329]

Strauss, John; Thomas, Duncan. Health over the life course. In: Schultz, TP.; Strauss, J., editors. Handbook of Development Economics. Vol. 4. Amsterdam: North Holland Press; 2008.

Winkleb MA, Jatulis EF, Fortmann SP. Socioeconomic Status and Health: How Education, Income and Occupation Contribute to Risk Factors for Cardiovascular Disease. American Journal of Public Health. Jun; 1992 82(6):816-820. [PubMed: 1585961]

Waite, Linda J.; Gallagher, Maggie. The Case for Marriage: Why Married People are Happier, Healthier, and Better Off Financially. New York: Doubleday; 2000.

Zhang X, Kanbur R. Spatial Inequality in Education and Health Care in China. China Economic Review. 2005:189-204.

Zhang J, Smith KR. Household Air Pollution from Coal and Biomass Fuels in China: Measurements, Health Impacts, and Interventions. Environmental Health Perspectives. Jun; 2007 115(6):848-855. [PubMed: 17589590] 


\section{Table 1}

Health and SES Variation across Geography in China

\begin{tabular}{|c|c|c|c|c|}
\hline & Mean & Median & Top 5 percentile & Bottom 5 percentiles \\
\hline SRH poor or very poor & 0.27 & 0.26 & 0.14 & 0.48 \\
\hline Any ADL difficulty & 0.08 & 0.07 & 0.02 & 0.18 \\
\hline Any IADL difficulty & 0.21 & 0.20 & 0.06 & 0.43 \\
\hline CES-D [0-13] & 8.23 & 8.19 & 4.71 & 12.10 \\
\hline Hypertension & 0.42 & 0.42 & 0.24 & 0.58 \\
\hline Weight(kg) & 59.4 & 59.5 & 51.9 & 67.6 \\
\hline Height $(\mathrm{cm})$ & 158.4 & 158.5 & 162.7 & 152.6 \\
\hline Years of Schooling & 5.38 & 5.17 & 9.23 & 2.59 \\
\hline Household PCE (000s) & 10.59 & 7.56 & 24.51 & 4.24 \\
\hline \multicolumn{5}{|c|}{ Across Community (450 community units) } \\
\hline & Mean & Median & Top 5 percentile & Bottom 5 percentiles \\
\hline SRH poor or very poor & 0.27 & 0.25 & 0.09 & 0.49 \\
\hline Any ADL difficulty & 0.08 & 0.07 & 0.00 & 0.23 \\
\hline Any IADL difficulty & 0.21 & 0.19 & 0.03 & 0.47 \\
\hline CES-D [0-13] & 8.20 & 8.00 & 4.16 & 12.66 \\
\hline Hypertension & 0.42 & 0.41 & 0.19 & 0.64 \\
\hline Weight(kg) & 59.5 & 59.16 & 50.3 & 68.2 \\
\hline Height(cm) & 158.4 & 158.5 & 163.6 & 152.9 \\
\hline Years of Schooling & 5.44 & 5.00 & 9.80 & 2.22 \\
\hline Household PCE (000s) & 11.01 & 7.21 & 29.29 & 3.69 \\
\hline
\end{tabular}

Source: Baseline CHARLS data 2011-2012. 
Table 3A

Percent Distribution of Human-made Environmental Attributes of Communities

\begin{tabular}{|c|c|c|c|}
\hline & Total & Urban & Rural \\
\hline \multicolumn{4}{|l|}{ Different drinking water types } \\
\hline Tap water (left-out group) & 51.9 & 80.7 & 26.8 \\
\hline Well water (Underground water) & 37.2 & 16.9 & 55.0 \\
\hline Pool water (Surface water) & 0.6 & 0 & 1.1 \\
\hline River \& lakes (Surface water) & 1.2 & 0.5 & 1.8 \\
\hline Cellar water (Surface water) & 1.2 & 0.4 & 1.9 \\
\hline Spring water (Underground water) & 0.8 & 0 & 1.4 \\
\hline Other & 5.9 & 1.2 & 10.1 \\
\hline \multicolumn{4}{|l|}{ Waste managements } \\
\hline Moved away by truck (left-out group) & 49.7 & 80.7 & 21.9 \\
\hline Buried in this village (Other) & 5.4 & 3.3 & 7.2 \\
\hline Burn away (Other) & 2.7 & 1.9 & 3.4 \\
\hline Put into nearby river (Other) & 0.7 & 0.5 & 0.8 \\
\hline Do not manage (Other) & 41.7 & 13.7 & 66.7 \\
\hline \multicolumn{4}{|l|}{ Toilet types } \\
\hline Inside toilet with water (Left-out Group & 43.4 & 69.8 & 19.8 \\
\hline Inside toilet without water (Without) & 3.3 & 3.8 & 3 \\
\hline Outside toilet with water (With) & 4 & 3.8 & 4.2 \\
\hline Outside public toilet without water (Without) & 9.1 & 9.9 & 8.4 \\
\hline Open air (Without) & 37 & 11.8 & 59.5 \\
\hline Others (With) & 3.1 & 0.9 & 5.1 \\
\hline \multicolumn{4}{|l|}{ Type of cooking fuels } \\
\hline Hay & 33.09 & 12.68 & 51.22 \\
\hline Coal & 13.11 & 10.83 & 15.13 \\
\hline Marsh gas & 2.93 & 0.65 & 4.96 \\
\hline Natural gas (Left-out group) & 17.14 & 32.42 & 3.57 \\
\hline LPG (Left-out group) & 28.17 & 37.98 & 19.46 \\
\hline Other & 5.56 & 5.45 & 5.66 \\
\hline Observations & 450 & 213 & 237 \\
\hline
\end{tabular}


Table 3B

Percent Distribution of Natural Environmental Attributes of Communities

\begin{tabular}{lccc}
\hline & Mean & Top 5 percentile & Bottom 5 percentile \\
\hline Lowest temperature & -8.28 & 6.00 & -30.00 \\
Highest temperature & 37.09 & 40.00 & 31.00 \\
Rainy days last year & 53.00 & 7.00 & 130.00 \\
Snowy days last year & 8.10 & 0.00 & 30.00 \\
Topography types distribution & Total & Urban & Rural \\
\cline { 2 - 4 } Plain & 45.09 & 59.43 & 32.20 \\
Hill & 27.23 & 19.81 & 33.90 \\
Mountainous region & 20.76 & 13.68 & 27.12 \\
Plateau & 4.46 & 3.77 & 5.08 \\
Basin & 2.46 & 3.30 & 1.69 \\
Observations & 450 & 213 & 237 \\
\hline
\end{tabular}

Data Source: CHARLS 2011-2012 Baseline 


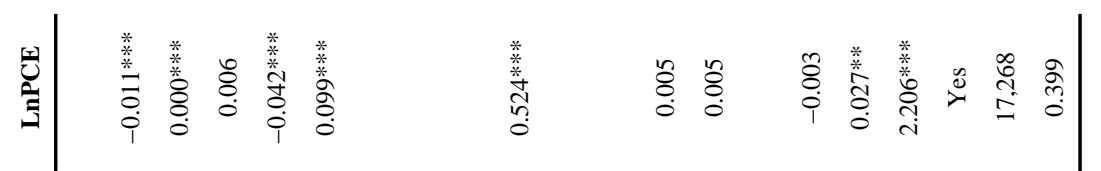

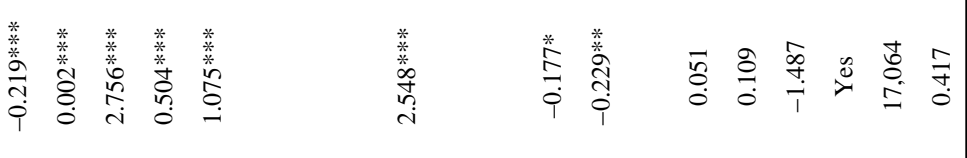

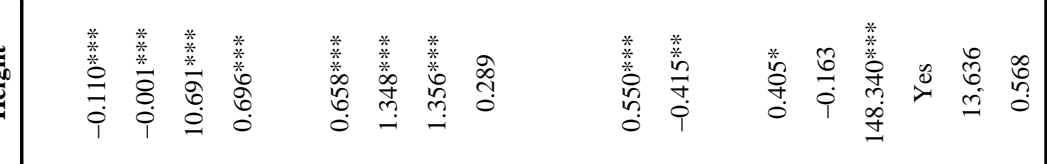

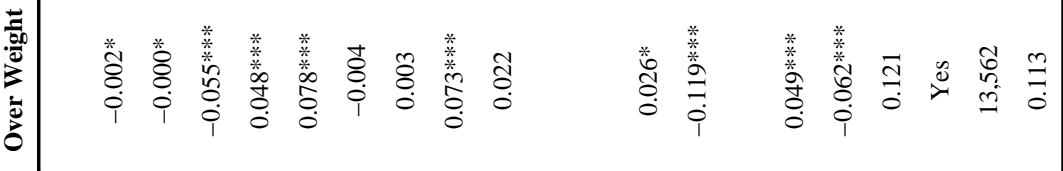

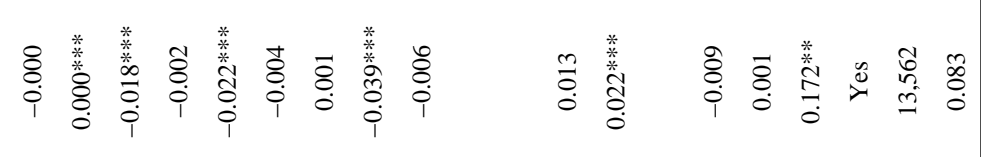

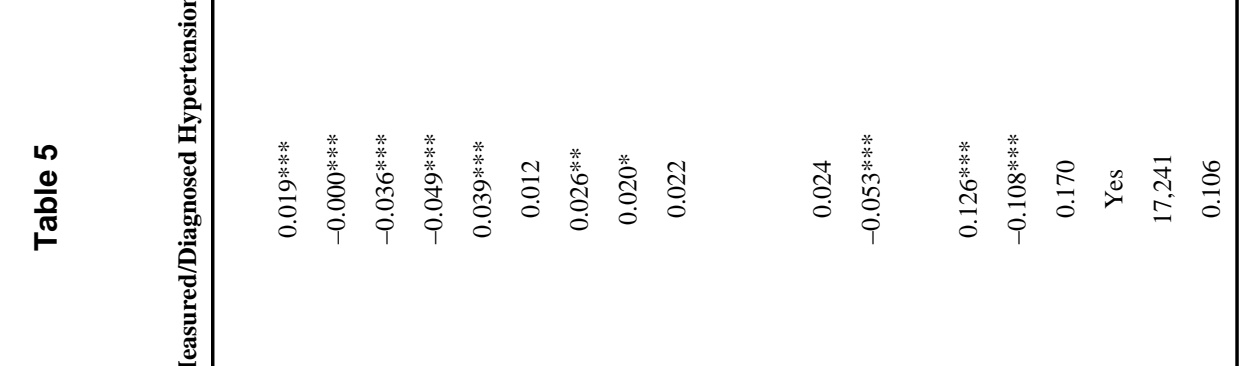




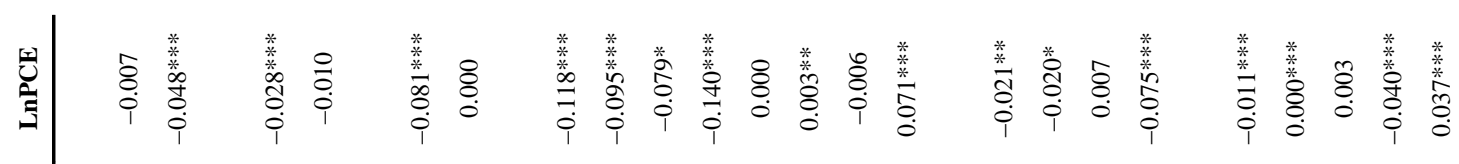

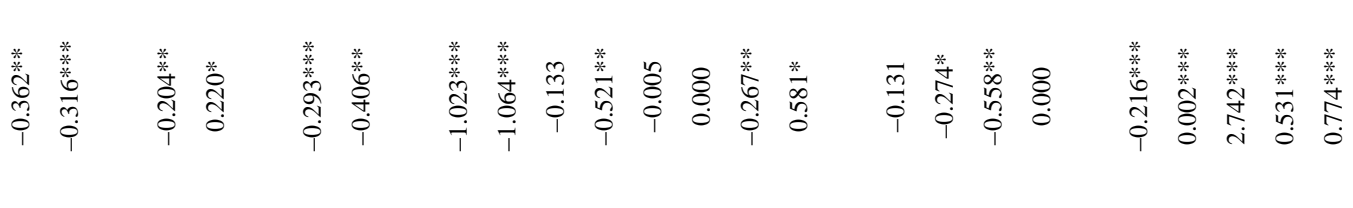

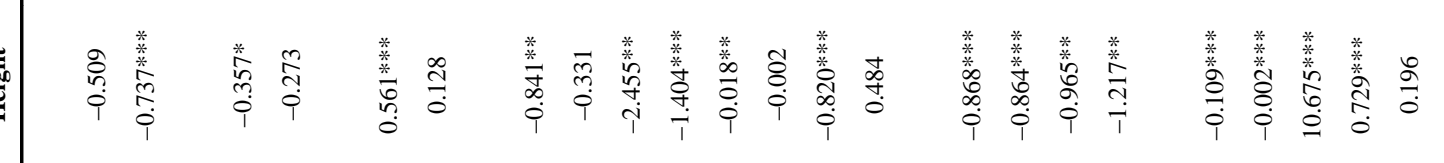

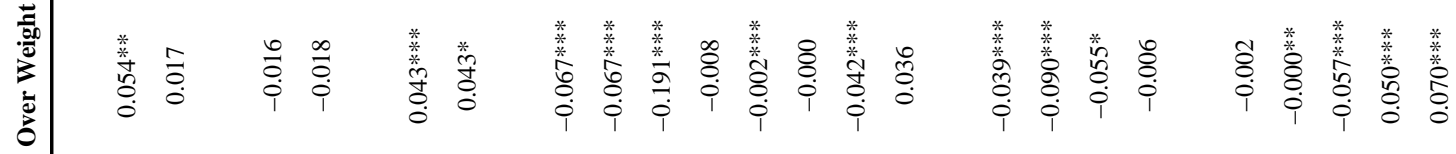

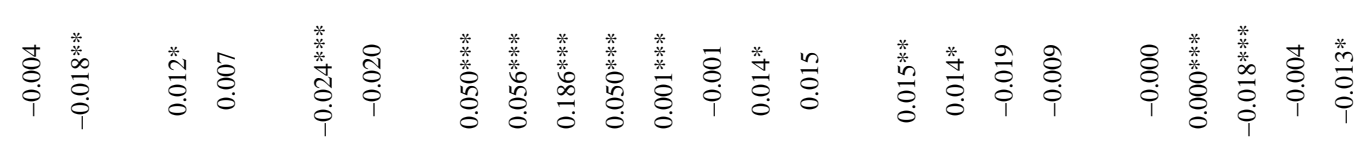

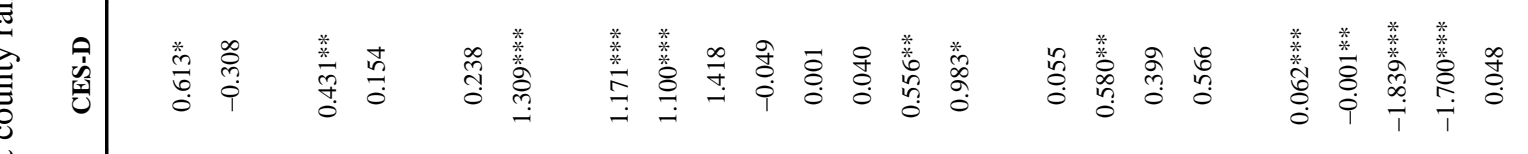

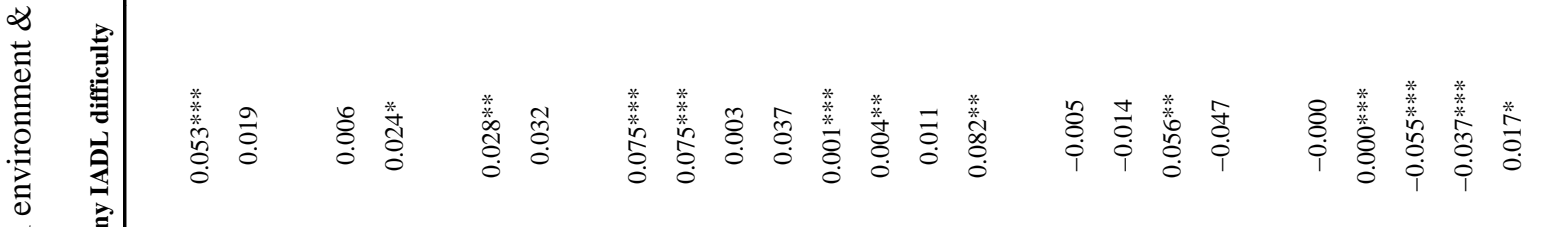

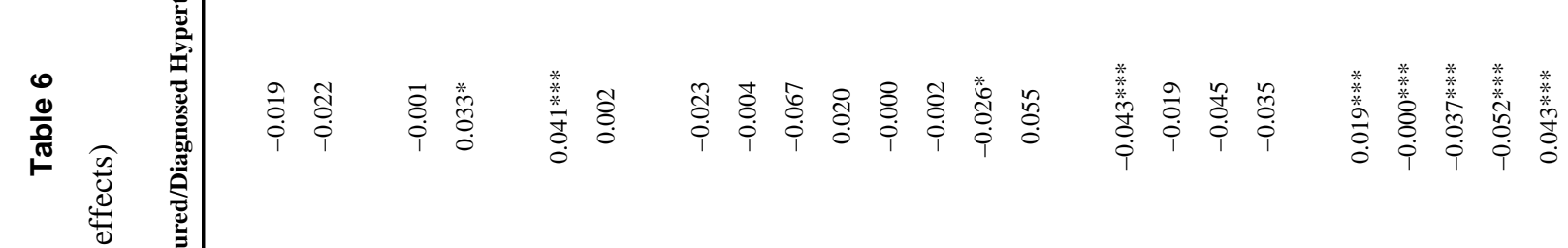


